\title{
Optimization Based Rate Control for Multicast with Network Coding: A Multipath Formulation
}

\author{
Tao Cui, Lijun Chen, and Tracey Ho
}

\begin{abstract}
Network coding offers new capabilities for efficient information multicasting in communication networks. In this paper, we study rate control for multicast with network coding. We propose a path-based multicasting scheme, where each source has multiple paths to each receiver in the multicast group. One advantage of this approach over a tree-based approach is that it is easier to find a minimum cost set of paths as compared to trees. We present three end-to-end rate control algorithms which operate over the set of paths supplied by the network layer. With random network coding, all algorithms can be implemented in a distributed manner. Our algorithms can also be generalized to solve other optimization problems with non-strictly concave objection function.
\end{abstract}

\section{INTRODUCTION}

Multicasting provides an efficient way of transmitting data from a sender to a group of receivers. The deployment of multicast involves at least two technical challenges. The first challenge involves multicast routing, i.e., the ability for a single source node to send information to multiple destinations at the same time. Network coding, proposed in [1], can significantly improve the throughput of existing network. Network coding extends the functionality of network nodes from storing/forwarding packets to performing algebraic operations on received data. The other challenge involves effective rate control, which is required to avoid congestion while ensuring high utilization and fair allocation of available resources among competing users.

In this paper, we address the problem of rate control for network coding based multicast with elastic rate demand. We can consider two general routing schemes over which end-to-end multicast rate control can be applied: tree-based and path-based routing. A tree-based multicast rate control scheme is presented in [2], where multiple multicast trees are used for multicasting and network coding is applied to flows across multicast trees. However, the issue of how to find a minimum cost set of multicast trees is not addressed. Relative to a tree-based approach, a path-based approach is less complex from an optimization standpoint, particularly since the criterion for whether network coding can occur across different tree branches/paths is whether they serve common sinks. We thus propose using a path-based scheme. By using the algorithms in, e.g., [3], we can find link disjoint paths from a source node to each of the destination nodes based on cost criteria that are independent of flow rates.

This work has been supported in part by DARPA grant N66001-06-C2020, Caltech's Lee Center for Advanced Networking, a gift from Microsoft Research, and NSF through grant CNS-0435520.

T. Cui, L. Chen, and T. Ho are with Division of Engineering and Applied Science, California Institute of Technology, Pasadena, CA, USA 91125. Email: \{taocui, tho\}ecaltech.edu, chenecds.caltech.edu
The main contribution of this paper is to present optimization model and propose stable, adaptive distributed rate control algorithms for multipath multicast with network coding. We extend the basic utility maximization formulation [4], [5] to the problem of multipath multicast with intrasession network coding. As the objective function is not strictly concave with respect to all the variables involved, "routing oscillation/instability" may occur in solving the relaxed problem and it may be difficult to recover the optimal values for those variables. We propose three adaptive rate control algorithms that overcome these challenges. The first one extends the primal variable recover algorithm for linear programming in [6]. The second one is based on primal-dual subgradient algorithm, where both primal and dual variables are updated using a subgradient algorithm. Similar to [2], the last algorithm combines rate control with the optimal routing in [7]. With random network coding, all algorithms can be implemented in a distributed manner, and are promising in practical implementation.

Related Work: Considerable research has been done on rate control for routing based multicast, see, e.g., [8], [9]. For network coding based multicast, Lun et. al. [10] proposed a dual subgradient method for finding the minimum cost multicast coding subgraph. Maximizing the difference between a utility and the total cost of coding subgraph is considered in [11]. In [12], a distributed algorithm for intrasession network coding and scheduling is proposed based on back-pressure. Utility maximization based rate control is studied in [2] for network coding based multicasting, where networks with and without given coding subgraphs are both considered. For multipath based unicast rate control, in [13], two end-to-end rate control algorithms are proposed. The first one modifies the objective function by adding a quadratic term while still preserving the same optimal solution. The second one shares some similarities with our combined rate control and adaptive routing algorithm. A similar approach to the first algorithm in [13] is adopted in [14], where convergence is rigorously proved. To our knowledge, rate control for multipath multicast either with network coding or without network coding has not been considered before. As will be shown later, this is not a straightforward extension of its unicast counterpart.

\section{Preliminaries}

\section{A. Network Model}

Consider a network $\mathcal{G}=(\mathcal{N}, \mathcal{L})$, with a set $\mathcal{N}$ of nodes and a set $\mathcal{L}$ of directed links. We denote a link either by a single index $l$ or by the directed pair $(i, j)$ of nodes it connects. Each link $l$ has fixed finite capacity $c_{l}$ bits per 


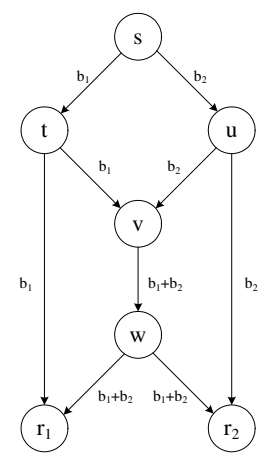

Fig. 1. The butterfly network.

second. A set of multicast sessions $\mathcal{M}$ is transmitted through the network. Each session $m \in \mathcal{M}$ is associated with one source $^{1} s_{m} \subset \mathcal{N}$ and a set $\mathcal{R}_{m} \subset \mathcal{N}$ of receivers. We consider unirate-multicast, where all receivers in session $m$ receive service at the same rate $x^{m}$ bits per second from $s_{m}$.

\section{B. Network Coding}

With network coding, each node is allowed to perform algebraic operations on received packets. We adopt the wellknown butterfly example in [1] as an illustrative example. In Fig. 1, without network coding, $s$ can only multicast 1 packet to $r_{1}$ and $r_{2}$ due to the bottleneck arc $(v, w)$. However, by using the transmission scheme shown in Fig. 1, two bits can be multicasted to $r_{1}$ and $r_{2}$. In this paper, we assume that coding is done only across packets of the same session. Define $f_{l}^{m r}$ as the information flow on link $l$ for receiver $r$ of session $m$. From the flow sharing property of network coding and the capacity constraint, we have

$$
\sum_{m \in \mathcal{M}} \max _{r \in \mathcal{R}_{m}} f_{l}^{m r} \leq c_{l}, \forall l \in \mathcal{L} .
$$

To obtain a fully distributed scheme, we use distributed random network coding [15], where for each node the data on outgoing links are random linear combination of the data on incoming links.

\section{Multipath Generation}

Internet congestion control has been dominated by end-toend schemes (in particular, TCP) [4], [5], primarily due to scalability and deployability as compared with hop-by-hop algorithms (e.g., back-pressure based algorithms in [2], [12]). Usually, end-to-end algorithms require that the network layer provides a minimum cost multicast tree (or Steiner tree) connecting the source to all the receivers in a multicast session. To find a minimum cost multicast tree is an NP-hard problem, though there exist many protocols for multicast tree construction such as distance vector multicast routing protocol [16]. Moreover, in order to exploit network coding opportunity, multiple multicast trees are required.

It is not clear how to choose a set of multiple multicast trees that is optimal for some cost function. First, the number of multicast trees in a network can be very large. For example, the simple network in Fig. 1 already contains

\footnotetext{
${ }^{1}$ Our algorithms can be extended to handle multiple source multicasting in a straightforward way.
}

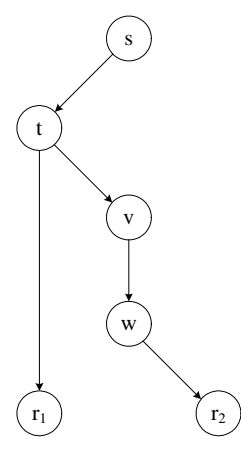

(a)

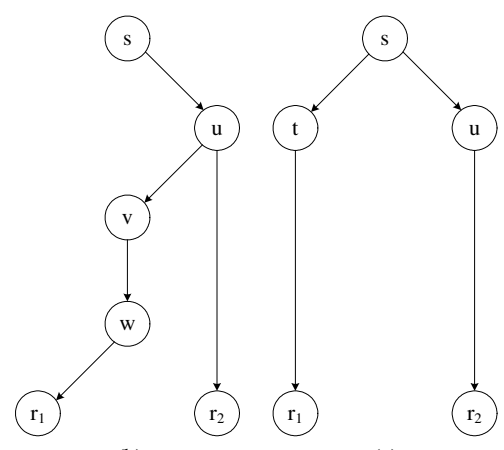

(b) (c)
Fig. 2. Multicast trees for the butterfly network shown in Fig. 1.

7 multicast trees. We only list three of them in Fig. 2. Second, the flow sharing relationship between different trees is complicated. Let $\mathcal{T}$ denote the set of all possible trees in a network and $\mathcal{R}_{l}^{T}$ denote the set of receivers that the packets on link $l$ in tree $T \in \mathcal{T}$ are intended for, e.g., $R_{(v, w)}^{T_{a}}=\left\{r_{2}\right\}$ for the tree in Fig. 2 (a). For any two trees $T_{1}$ and $T_{2}$ in $\mathcal{T}$, if link $l \in T_{1} \cap T_{2}$, the flows on $T_{1}$ and $T_{2}$ can be coded together on link $l$ if and only if $\mathcal{R}_{l}^{T_{1}} \cap \mathcal{R}_{l}^{T_{2}}=\emptyset$ or $T_{1}$ and $T_{2}$ have disjoint sets of downstream destinations, which is also the condition for the algorithm in [2] to work. For example, in Fig. 2, if we consider $T_{a}$ and $T_{c}, \mathcal{R}_{\left(t, r_{1}\right)}^{T_{a}}=\left\{r_{1}\right\}$, $\mathcal{R}_{\left(t, r_{1}\right)}^{T_{c}}=\left\{r_{1}\right\}$, and $\mathcal{R}_{\left(t, r_{1}\right)}^{T_{a}} \cap \mathcal{R}_{\left(t, r_{1}\right)}^{T_{c}}=\left\{r_{1}\right\}$. Therefore, we cannot mix the packets from $T_{a}$ and $T_{c}$ on link $\left(t, r_{1}\right)$, and $T_{a}$ and $T_{b}$ cannot coexist (or if they can coexist, the rate control algorithm will be complicated.). In a large network, it is complicated to check whether two trees share any links and whether $\mathcal{R}_{l}^{T_{1}} \cap \mathcal{R}_{l}^{T_{2}}=\emptyset$ is satisfied on these links.

Note that efficient network coding relies on, first, the existence of a set of link disjoint paths for each sourcereceiver pair and, second, that the sets of paths to different receivers should share some common links. The former condition provides higher capacity than a single path to each receiver, while the latter provides the network coding opportunities. So, taking a multipath approach, we find $h$ link disjoint paths from each source node to its corresponding destination nodes based on cost criteria that are independent of flow rates, by using techniques such as from [3]. In the following, we will restrict our attention to rate control in transport layer assuming multiple paths are given.

\section{Multipath Multicast Rate Control}

\section{A. Problem Formulation}

We study rate control in utility maximization framework. Let $\mathcal{P}^{m r}$ be the set of paths used by receiver $r$ in session $m$. We are interested in finding the optimal traffic rates on these paths. For any path $p \in \mathcal{P}^{m r}$, let the set of links on the path be denoted by $\mathcal{L}_{p}$. For each receiver $r \in \mathcal{R}_{m}$, we associate it with a $|\mathcal{L}| \times\left|\mathcal{P}^{m r}\right|$ multipath matrix $\mathbf{H}^{m r}$ whose $(l, p)$ th entry is given by: $H_{l p}^{m r}=1$ if $l \in \mathcal{L}_{p}$ and $H_{l p}^{m r}=$ 0 otherwise. As the paths are link disjoint, every row in $\mathbf{H}^{m r}$ contains at most one 1 . Denote by $x_{p}^{m r}$ the rate at path $p \in \mathcal{P}^{m r}$. Since we only consider unirate multicast, we have $\sum_{p \in \mathcal{P}^{m r}} x_{p}^{m r}=x^{m}, \forall r \in \mathcal{R}_{m}$, where $x^{m}$ is the multicast 
rate of session $m$. With intra-session network coding, the link capacity constraint (1) becomes

$$
\sum_{m \in \mathcal{M}} \max _{r \in \mathcal{R}_{m}}\left\{\sum_{p \in \mathcal{P}^{m r}} H_{l p}^{m r} x_{p}^{m r}\right\} \leq c_{l}, \forall l \in \mathcal{L} .
$$

Following [4], we assume each session $m$ attains a utility $U_{m}\left(x^{m}\right)$, where $U_{m}(\cdot)$ is continuously differentiable, increasing, and strictly concave. Our objective is to maximize the aggregate utilities over all the sessions. The problem can be posed as

$$
\begin{aligned}
\max _{x} & \sum_{m \in \mathcal{M}} U_{m}\left(x^{m}\right) \\
\text { subject to } & \sum_{p} H_{l p}^{m r} x_{p}^{m r} \leq y_{l}^{m}, \forall r \in \mathcal{R}_{m}, \\
& \sum_{m \in \mathcal{M}} y_{l}^{m} \leq c_{l}, \forall l \in \mathcal{L}, \\
& \sum_{p \in \mathcal{P}^{m r}} x_{p}^{m r}=x^{m}, \forall m \in \mathcal{M}, \forall r \in \mathcal{R}_{m},
\end{aligned}
$$

where by introducing auxiliary variables $y_{l}^{m}$ we replace the constraint (2) with the first two linear inequalities in (3). $y_{l}^{m}$ can be interpreted as the physical flow rate of session $m$ on link $l$. Note that the last set of constraints in (3) contains $\left|\mathcal{R}_{m}\right|$ equalities, while that in the multipath rate control for unicast sessions in [13], [14] only contains a single equality constraint. In [13], [14], the authors do not write out $x^{m}$ explicitly, but instead discard the equality and write the utility as a function of path rate directly. This approach does not extend to the multicast case as we cannot simply discard $x^{m}$ in (3). On contrary, all proposed algorithms in the following can be used for the unicast case.

\section{B. Primal Recovery Algorithm}

To proceed, due to the difficulty brought in by the set of equality constraints in (3), we first rewrite (3) as

$$
\begin{aligned}
\max _{x} & \sum_{m \in \mathcal{M}} U_{m}\left(x^{m}\right)-\beta_{m} \sum_{r \in \mathcal{R}_{m}}\left(x^{m}-\sum_{p \in \mathcal{P}^{m r}} x_{p}^{m r}\right)^{2} \\
\text { subject to } & \sum_{p \in \mathcal{P}^{m r}} H_{l p}^{m r} x_{p}^{m r} \leq y_{l}^{m}, \forall r \in \mathcal{R}_{m} \\
& \sum_{m \in \mathcal{M}} y_{l}^{m} \leq c_{l}, \forall l \in \mathcal{L}, \\
& x^{m} \leq \sum_{p \in \mathcal{P}^{m r}} x_{p}^{m r}, \forall m \in \mathcal{M}, \forall r \in \mathcal{R}_{m}
\end{aligned}
$$

where $\beta_{m}>0$ is a positive constant. It is easy to show that (4) is equivalent to (3). As the objective function in (4) is not strictly concave in $x_{p}^{m r}$, all dual based algorithms in [4], [5] have the same oscillation problem. Both [13], [14] attempt to address this problem by introducing an auxiliary variable and adding a quadratic term onto the objective function for each $x_{p}^{m r}$. Rate control algorithms in [13], [14] can also be used to solve (4). But in the multicast case, the total number of auxiliary variables is equal to the total number of receivers in all the multicast sessions, which may be very large, and makes rate control complicated. Instead of introducing auxiliary variables, we solve (4) directly.

The dual function obtained by relaxing only the first and the last sets of constraints in (4) can be further decomposed into two subproblems

$$
\begin{gathered}
\phi_{1}(q, \lambda)=\max _{x} \sum_{m} U_{m}\left(x^{m}\right)-\beta_{m} \sum_{r}\left(x^{m}-\sum_{p} x_{p}^{m r}\right)^{2} \\
-\sum_{m, r, p}\left(\sum_{l} q_{l}^{m r} H_{l p}^{m r}\right) x_{p}^{m r}-\sum_{m, r} \lambda^{m r}\left(x^{m}-\sum_{p} x_{p}^{m r}\right), \\
\phi_{2}(q)=\max _{y} \sum_{l, m}\left(\sum_{r} q_{l}^{m r}\right) y_{l}^{m} \\
\quad \text { subject to } \sum_{m} y_{l}^{m} \leq c_{l}, \forall l \in \mathcal{L},
\end{gathered}
$$

where $q_{l}^{m r}$ is the Lagrange multiplier at link $l$ for receiver $r$ in session $m$, and $\lambda^{m r}$ is the Lagrange multiplier for receiver $r$ in session $m . q_{l}^{m r}$ can be interpreted as the "congestion price" at link $l$ for receiver $r$ in session $m$, which is charged by link $l$ for transmitting unit bit rate. $\lambda^{m r}$ can be interpreted as the "rate bonus" at source $s^{m}$, which is awarded by sending unit bit rate for receiver $r$ in session $m$. The first subproblem (5) is multipath multicast rate control at each source. We need to split the multicast rate among multiple paths for each receiver. The second subproblem (6) is joint network coding and session scheduling. The two subproblems interact through $p$ and $\lambda$.

Multicast rate control: To solve (5), we first fix $x^{m}$ and optimize over $x_{p}^{m r}$. Given dual variables $q_{l}^{m r}(t)$ and $\lambda^{m r}(t)$ at time $t$, solving (5) for $x_{p}^{m r}$ is equivalent to solving

$$
\min _{x_{p}^{m r}} \beta_{m}\left(x^{m}-\sum_{p} x_{p}^{m r}\right)^{2}+\sum_{p}\left(\sum_{l} q_{l}^{m r} H_{l p}^{m r}-\lambda^{m r}\right) x_{p}^{m r} .
$$

Let $\hat{p}^{m r}(t)$ be the path with minimum $\sum_{l} q_{l}^{m r}(t) H_{l p}^{m r}$ at time $t$, i.e., $\hat{p}^{m r}(t)=\arg \min _{p} \sum_{l} q_{l}^{m r}(t) H_{l p}^{m r}$, and $w^{m r}(t)=\min _{p} \sum_{l} q_{l}^{m r}(t) H_{l p}^{m r}$ be its path cost at time $t$. The optimal solution of (7) can be obtained as

$x_{p}^{m r}(t+1)=\left\{\begin{array}{cc}\max \left\{x^{m}-\frac{w^{m r}(t)-\lambda^{m r}(t)}{2 \beta_{m}}, 0\right\}, & \text { if } p=\hat{p}^{m r}, \\ 0, & \text { otherwise. }\end{array}\right.$

To see why (8) is correct, if we transmit packets at rate $x^{\prime}$ on a non-minimum price path $p^{\prime}$, we can always shift the rate $x^{\prime}$ to the minimum price path to make the objective function of (7) smaller. Therefore each source should only transmit packets on the path with the minimum price to each receiver.

Let $x^{m}(t)$ and $x^{m}(t+1)$ denote the source rates at time $t$ and $t+1$ respectively. Substituting (8) into (5) and optimizing over $x^{m}$, we obtain

$\quad x^{m}(t+1)=\underset{x \geq 0}{\arg \max } U_{m}(x)-\sum_{r} f^{m r}(x)$,
where
\[ f^{m r}(x)=\left\{\begin{array}{cc}\beta_{m} x^{2}+\lambda^{m r}(t) x, & \text { if } x \leq \frac{w^{m r}(t)-\lambda^{m r}(t)}{2 \beta_{m}^{m} m r}, \\ w^{m r}(t) x, & \text { if } x>\frac{w^{m r}(t)-\lambda^{m r}(t)}{2 \beta_{m}} .\end{array}\right. \]

Path rate adaptation: If we adopt (8) for path rate directly, this clearly introduces undesirable rate oscillation among different paths. This rate oscillation is because the objective function in (3) is not strictly concave in $x_{p}^{m r}$. One way to solve the rate oscillation problem is to adopt the primal recovery algorithm in [6] for linear programming. We extend this algorithm to solve our convex optimization problem (3). To recover the multicast rate $x^{m}$ and path rate $x_{p}^{m r}$, we compose primal iterates of $\tilde{x}^{m}(t+1)$ and $\tilde{x}_{p}^{m r}(t+1)$ via

$\tilde{x}^{m}(t+1)=\sum_{\tau=1}^{t+1} \mu_{\tau}^{t+1} x^{m}(\tau), \tilde{x}_{p}^{m r}(t+1)=\sum_{\tau=1}^{t+1} \mu_{\tau}^{t+1} x_{p}^{m r}(\tau)$, 
where $\mu_{\tau}^{t+1}$ is the weight of $x_{p}^{m r}(\tau), \sum_{\tau=1}^{t+1} \mu_{\tau}^{t+1}=1$, and $\mu_{\tau}^{t+1} \geq 0$. Conditions on $\mu_{\tau}^{t+1}$ to guarantee the convergence of our primal-recovery algorithm will be given later.

Network coding and session scheduling: At time $t+1$, we solve (6) given congestion price $q_{l}^{m r}(t)$. For each link $l$, let $\hat{m}_{l}(t+1)=\arg \max _{m} \sum_{r} q_{l}^{m r}(t)$ be the multicast session with the maximum aggregate link price. The solution of (6) is given by the following assignment

$$
y_{l}^{m}(t+1)=\left\{\begin{array}{cc}
c_{l}, & \text { if } m=\hat{m}_{l}(t+1), \\
0, & \text { otherwise. }
\end{array}\right.
$$

Mathematically, this is because (6) is a linear programming and we can always choose an extreme point solution.

Random linear network coding scheme is used to code packets from the same multicast session. For each link $l$, coded packets for session $\hat{m}_{l}(t+1)$ is sent at the rate of $c_{l}$.

Dual variable update: By using the subgradient method, each link $l$ updates its congestion price with respect to receiver $r$ in session $m$ according to

$$
q_{l}^{m r}(t+1)=\left[q_{l}^{m r}(t)-\gamma_{t}\left(y_{l}^{m}(t)-\sum_{p} H_{l p}^{m r} x_{p}^{m r}(t)\right)\right]^{+},
$$

and each source $s^{m}$ updates $\lambda^{m r}$ for receiver $r$ in session $m$ according to

$$
\lambda^{m r}(t+1)=\left[\lambda^{m r}(t)-\gamma_{t}\left(\sum_{p} x_{p}^{m r}(t)-x^{m}(t)\right)\right]^{+},
$$

where $\gamma_{t}$ is a positive scalar stepsize and ' + ' denotes the projection onto the set $\mathbb{R}^{+}$of non-negative real numbers. Note that we use $x^{m}$ and $x_{p}^{m r}$ from (9) and (8) in (13) and (14) instead of using (11), which can be implemented by putting the values of (9) and (8) in the header of each packet and update dual variables using this header instead of instantaneous rate on each link. Moreover, (13) can be implemented at individual links using only local information, i.e., $H_{l p}^{m r} x_{p}^{m r}$ and $y_{l}^{m}$. The same is also true for (14). We can also use $x^{m}$ and $x_{p}^{m r}$ from (11) to update dual variables. However, its convergence is slow. But this approach can be combined with our primal recovery algorithm. The primal recovery algorithm is run first and after a certain number of steps it updates dual variables by using (11).

If we assume that $\gamma_{t}$ is diminishing in (13) and (14) and that $\gamma_{t}$ and $\mu_{\tau}^{t+1}$ satisfy the same conditions as those given in [6], by following the proof in [6] we can show that the accumulation points of $\left\{\tilde{x}^{m}(t)\right\}$ and $\left\{\tilde{x}_{p}^{m r}(t)\right\}$ are optimal solutions to (4). However, in network rate control, we prefer a constant stepsize $\gamma$ in the dual update.

Define $\rho(x, \mathcal{S})=\min _{y \in \mathcal{S}}\|x-y\|$ as the Euclidean distance of a point $x$ to set $\mathcal{S}$, and $\Phi_{r}(\mathcal{S})$ be the set of all points at a distance of $r$ or less from $S$, i.e., $\Phi_{r}(\mathcal{S})=\{x$ : $\rho(x, \mathcal{S}) \leq r\}$. Also, let $\mathcal{X}^{*}$ be the set of optimal solutions of (4). Let $\Delta_{t} \triangleq \max _{1 \leq \tau \leq t} \mu_{\tau}^{t}-\mu_{\tau-1}^{t}$, and $\gamma_{t}=\gamma$ be the constant stepsize. The following theorem shows the convergence of primal recovery algorithm with constant stepsize.

Theorem 1: Let $\{\vec{x}(t)\}$ be the sequence of rate vectors defined by $\left\{\left[\tilde{x}^{m}(t), \tilde{x}_{p}^{m r}(t)\right]\right\}$. If the convex combination weights $\mu_{\tau}^{t}, \forall \tau, t$, are chosen to satisfy:

1) $\mu_{\tau}^{t} \geq \mu_{\tau-1}^{t}, \forall t$ and $2 \leq \tau \leq t$,
2) $\lim \sup _{t \rightarrow+\infty} t \Delta_{t} \leq \delta$ for some constant $\delta \geq 0$,

3) $\mu_{1}^{t} \rightarrow 0$ as $t \rightarrow+\infty$,

then there exists a function $r(\gamma, \delta)$ such that

$$
\lim _{t \rightarrow+\infty} \rho\left(\vec{x}(t), \Phi_{r(\gamma, \delta)}\left(\mathcal{X}^{*}\right)\right)=0, \forall \gamma>0 .
$$

Furthermore, if $\delta=0, \lim _{\gamma \rightarrow 0} r(\gamma, \delta)=0$.

Theorem 1 can be proved by using Lyapunov method. The detailed proof can be found in [17]. Theorem 1 states that for a constant stepsize, $\vec{x}(t)$ converges to a neighborhood around the optimum, and the size of this neighborhood becomes arbitrarily small with decreasing stepsize. From Theorem 1, one possible choice of $\mu_{\tau}^{t}$ is $1 / t$. One practical issue is that the first few $x^{m}(t)$ and $x_{p}^{m r}(t)$ may not be good. To accelerate the algorithm, we only average $x^{m}(t)$ and $x_{p}^{m r}(t)$ from time $T$ in (11). We can show that Theorem 1 still holds in this case.

\section{Primal-Dual Subgradient Algorithm}

In this subsection, we also reformulate the rate control problem as (4). The multicast rate control, network coding and session scheduling, and dual variable update components of this algorithm are identical to those in Section III-B. The only difference is that we apply primal-dual subgradient algorithm in the path rate adaptation to resolve the rate oscillation problem in (4) with direct dual based algorithm.

Multicast rate control: Different from Section III-B, we substitute path rates $x_{p}^{m r}(t)$ at time $t$ into (5) and optimize over $x^{m}$ first. In this case, the source $s^{m}$ adjusts its multicast rate $x^{m}$ according to the aggregate dual variables $\sum_{r} \lambda^{m r}$ over all the receivers and the aggregate path rates $\sum_{p} x_{p}^{m r}(t)$ for each receiver in $\mathcal{R}_{m}$,

$$
\begin{aligned}
x^{m}(t+1)=\underset{x \geq 0}{\arg \max } & U_{m}(x)-\left(\sum_{r} \lambda^{m r}(t)\right) x \\
& -\beta_{m} \sum_{r}\left(x-\sum_{p} x_{p}^{m r}(t)\right)^{2} .
\end{aligned}
$$

Specifically, if $U_{m}(x)=\log x$, we have

$$
\begin{aligned}
x^{m}(t+1)= & \frac{\sqrt{\left(2 \beta_{m} \sum_{r, p} x_{p}^{m r}(t)-\sum_{r} \lambda^{m r}(t)\right)^{2}+8 \beta_{m}\left|\mathcal{R}_{m}\right|}}{4 \beta_{m}\left|\mathcal{R}_{m}\right|} \\
& +\frac{\beta_{m} \sum_{r, p} x_{p}^{m r}(t)-\sum_{r} \lambda^{m r}(t)}{4 \beta_{m}\left|\mathcal{R}_{m}\right|} .
\end{aligned}
$$

Note that both $x_{p}^{m r}(t)$ and $\lambda^{m r}(t)$ are known at the source. Thus $x^{m}(t+1)$ can be computed locally at the source.

Path rate adaptation: Instead of solving (5) directly, we update the path rate using a primal subgradient algorithm. At source $s^{m}$, each path $p$ of receiver $r$ in session $m$ updates its rate according to

$$
\begin{aligned}
x_{p}^{m r}(t+1)=\left[x_{p}^{m r}(t)-\right. & \epsilon_{t}\left(2 \beta_{m}\left(\sum_{p} x_{p}^{m r}(t)-x^{m}(t)\right)\right. \\
+ & \left.\left.\sum_{l} q_{l}^{m r}(t) H_{l p}^{m r}-\lambda^{m r}(t)\right)\right]^{+},
\end{aligned}
$$

where $\epsilon_{t}$ is a positive stepsize. Eq. (18) says that if the aggregate path rate $\sum_{p} x_{p}^{m r}$ exceeds multicast rate $x^{m}$ and 
$\sum_{l} q_{l}^{m r} H_{l p}^{m r}=\lambda^{m r}$, path rate $x_{p}^{m r}$ decreases, and increases otherwise. If aggregate price $\sum_{l} q_{l}^{m r} H_{l p}^{m r}$ on path $p$ of receiver $r$ is greater than rate bonus $\lambda^{m r}$ of $r$ and $\sum_{p} x_{p}^{m r}=$ $x^{m}$, path rate $x_{p}^{m r}$ also decreases, and increases otherwise. At the equilibrium, $\sum_{p} x_{p}^{m r}=x^{m}$ and $\sum_{l} q_{l}^{m r} H_{l p}^{m r}=\lambda^{m r}$, where the later condition implies that the cost of a path should be equal to the bonus obtained by using it.

In fact, (18) bears similarity with (11) in the primalrecovery algorithm. We can write (18) as a weighted sum of $x_{p}^{m r}(t)$ and $\check{x}_{p}^{m r}(t+1)=x^{m}(t)-\frac{\sum_{l} q_{l}^{m r}(t) H_{l p}^{m r}-\lambda^{m r}(t)}{2 \beta_{m}}$. Note that $\check{x}_{p}^{m r}(t+1)$ is similar to $x_{p}^{m r}(t+1)$ in $(8) . \check{x}_{p}^{m r}(t+1)$ is non-zero for all $p \in \mathcal{P}^{m r}$ while $x_{p}^{m r}(t+1)$ in (8) is non-zero only for the minimum cost path. Compared with (11), we can see that both algorithms use time-average mechanism to smooth the solution (8) returned by solving primal problem directly. In fact, the rate control algorithm in [14] is a special case of the primal-dual subgradient method, where the primal subgradient algorithm is applied to the auxiliary variables.

Theorem 2: The primal-dual subgradient algorithm (16)(18) converges to the optimal solution of problem (4) with diminishing stepsize.

The proof can be found in [17]. For a constant stepsize, the primal-dual subgradient method can be made to converge within any given small neighborhood around the optimum, by choose sufficiently small constant stepsize.

Instead of adding a quadratic penalty term in (4), we can write the dual of (3) by relaxing the equality constraints directly. Primal recovery and primal-dual subgradient can also be applied. However, our experimental results show that this formulation does not have good convergence properties.

\section{Combination of Rate Control and Optimal Routing}

In this subsection, we solve (3) directly instead of introducing penalty function. The dual problem to (3) by relaxing only the first set of constraints in (3) can be decomposed into two subproblems

$$
\begin{aligned}
\phi_{1}(q)= & \max _{x, \alpha} \sum_{m} U_{m}\left(x^{m}\right)-\sum_{l, m, r, p} q_{l}^{m r} H_{l p}^{m r} x_{p}^{m r} \\
& \text { subject to } \sum_{p} x_{p}^{m r}=x^{m}, x_{p}^{m r} \geq 0, \\
\phi_{2}(q)= & \max _{y} \sum_{l, m}\left(\sum_{r} q_{l}^{m r}\right) y_{l}^{m}, \text { subject to } \sum_{m} y_{l}^{m} \leq c_{l} .
\end{aligned}
$$

where $q_{l}^{m r}$ is Lagrange multiplier introduced at link $l$ for path $p$ of receiver $r$ in session $m$. As in Section III-B $q_{l}^{m r}$ can also be interpreted as the "congestion price". Again, (19) and (20) correspond to multipath multicast rate control, and joint network coding and session scheduling, respectively. Solving (20) is similar to solving (5) in Section III-B. In the following, we will restrict our attention to solving (19).

To solve (19), we first fix $x^{m}$ and optimize over $x_{p}^{m r}$, which is equivalent to solving

$$
\begin{aligned}
\min _{x} & \sum_{p}\left(\sum_{l} q_{l}^{m r} H_{l p}^{m r}\right) x_{p}^{m r}, \\
\text { subject to } & \sum_{p} x_{p}^{m r}=x^{m}, x_{p}^{m r} \geq 0, \forall p \in \mathcal{P}^{m r} .
\end{aligned}
$$

Let $\hat{p}^{m r}=\arg \min _{p} \sum_{l} q_{l}^{m r} H_{l p}^{m r}$ be the path with the minimum price for receiver $r$ in session $m$ (tie is broken arbitrarily). The optimal solution of (21) can be obtained as

$$
\hat{x}_{p}^{m r}=\left\{\begin{array}{cc}
x^{m}, & \text { if } p=\hat{p}^{m r}, \\
0, & \text { otherwise. }
\end{array}\right.
$$

The solution (22) corresponds to an extreme point in the polytope defined by $\sum_{p} x_{p}^{m r}=x^{m}, x_{p}^{m r} \geq 0$. Let $w^{m r}(t)=$ $\min _{p} \sum_{l} q_{l}^{m r}(t) H_{l p}^{m r}$. Denote $x^{m}(t)$ as the multicast rate of source $s^{m}$ at time $t$. We substitute (22) into (19) and optimize over $x^{m}$. As the utility function is strictly concave, we find that $x^{m}$ is adjusted according to $\sum_{r} w^{m r}(t)$ as

$$
x^{m}(t+1)=\left(U_{m}^{\prime}\right)^{-1}\left(\sum_{r} w^{m r}(t)\right) .
$$

Note that (23) is the optimal solution to (19). We can also update $x^{m}$ by using the primal subgradient algorithm according to $\sum_{r} w^{m r}(t)$.

Note that we cannot apply the path adaptation algorithms in Sections IV-A, B as we do not relax the equality constraint as in (4). Given $x^{m},(21)$ is a minimum cost optimal routing problem, which is fully studied in [7]. As in [7], we can eliminate the equality constraint $\sum_{p} x_{p}^{m r}=x^{m}$ in (21) by expressing the rate of path $\hat{p}^{m r}$ in terms of other path rates, i.e., $x_{\hat{p}^{m r}}^{m r}=x^{m}-\sum_{p \neq \hat{p}^{m r}} x_{p}^{m r}$. We can then write (21) as

$$
\begin{aligned}
& \min _{x} \sum_{p \neq \hat{p}^{m r}}\left(\sum_{l} q_{l}^{m r} H_{l p}^{m r}-w^{m r}\right) x_{p}^{m r}, \\
& \text { subject to } x_{p}^{m r} \geq 0, \forall p \in \mathcal{P}^{m r}, p \neq \hat{p}^{m r} .
\end{aligned}
$$

As in Section III-C, the flow rates $x_{p}^{m r}$ on paths other than the minimum cost path are updated using the primalsubgradient algorithm. The flow rate of the minimum cost path is then updated to satisfy the equality constraint in (21). For each receiver $r$ in session $m$, the source in session $m$ updates $x_{p}^{m r}$ according to

$$
\begin{aligned}
& x_{p}^{m r}(t+1)= \\
& \left\{\begin{array}{c}
{\left[x_{p}^{m r}(t)-\kappa_{t}\left(\sum_{l} q_{l}^{m r}(t) H_{l p}^{m r}-w^{m r}(t)\right)\right]^{+}, \text {if } p \neq \hat{p}^{m r}} \\
{\left[x^{m}(t+1)-\sum_{p \neq \hat{p}^{m r}} x_{p}^{m r}(t+1)\right]^{+}, \quad \text { otherwise }}
\end{array}\right.
\end{aligned}
$$

where $\kappa_{t}$ is a positive scalar stepsize and $\sum_{l} q_{l}^{m r}(t) H_{l p}^{m r}-$ $w^{m r}(t)$ is the current subgradient of $x_{p}^{m r}$. Thus, the flow rates on paths other than the minimum cost path are reduced by an amount proportional to the price difference $\sum_{l} q_{l}^{m r}(t) H_{l p}^{m r}-w^{m r}(t)$, and the flow rate on the minimum cost path is increased so that the new path rates of each receiver sum to the new multicast rate obtained by the multicast rate control algorithm (23). For each sourcereceiver pair, the path rate update algorithm (25) is similar to the optimal routing algorithm in [7]. Our algorithm is thus an integration of multicast rate control and optimal routing. When only unicast sessions exist, it reduces to Algorithm 2 in [13].

The underlying mechanism of all three algorithms is "primal average", where non-smooth primal variables are smoothed by using time average. We believe that the idea of primal average is general enough to solve a class of optimization problems with non-strict objective functions. Both primal recovery and primal-dual subgradient algorithms 


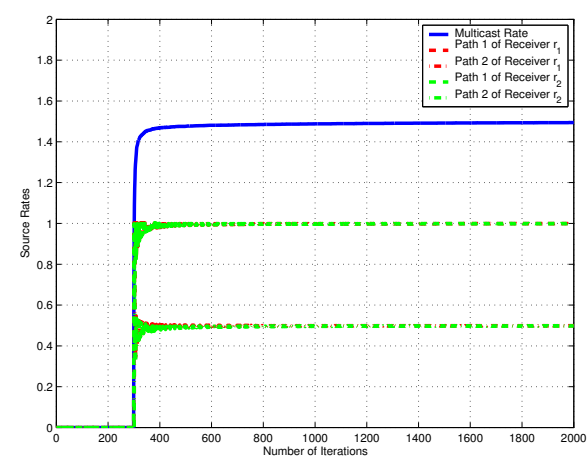

(a)

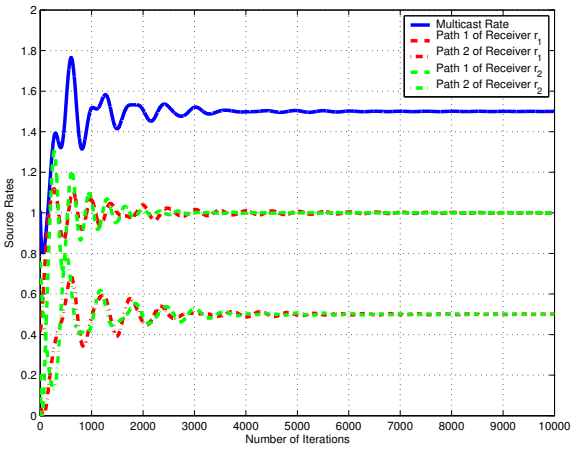

(b)

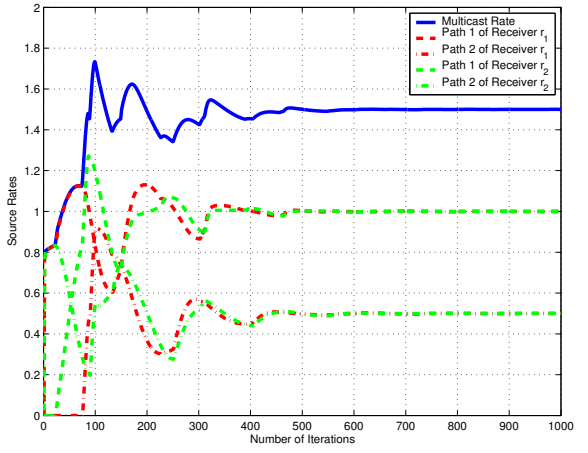

(c)

Fig. 3. The evolution of source rates versus the number of iterations for the butterfly network in Fig. 1: (a) The primal recovery algorithm with stepsizes $\gamma=0.01$ and $\beta=2$; (b) The primal-dual subgradient algorithm with stepsizes $\gamma=0.01$ and $\beta=0.5$; (c) The combination of rate control and optimal routing algorithm with stepsizes $\gamma=0.01$ and $\kappa=0.05$.

do not require that the utility function is strictly concave. But rate control with optimal routing does require strictly concave utility functions.

\section{NUMERICAL EXAMPLES}

We consider the network shown in Fig. 1, where all links have unit capacity except for link $(s, u)$ with a capacity 0.5 . There are only one multicast session with one source node $s$ and two receivers $r_{1}$ and $r_{2}$. The source attains a utility $U_{m}\left(x^{m}\right)=\log \left(x^{m}\right)$. Two link disjoint paths exist for each receiver: $\left\{(s, t),\left(t, r_{1}\right)\right\}$ and $\left\{(s, u),(u, v),(v, w),\left(w, r_{1}\right)\right\}$ for $r_{1},\left\{(s, t),(t, v),(v, w),\left(w, r_{2}\right)\right\}$ and $\left\{(s, u),\left(u, r_{1}\right)\right\}$ for $r_{2}$.

Fig. 3 shows the evolution of source rates by using different algorithms. In Fig. 3 (a), we choose $\mu_{\tau}^{t}=\frac{1}{t-T+1}$ in (11) with $T=300$, which means that we only apply the primal average operations after 300 iterations. We observe that all rates converge within a small neighborhood of the optimal values as we have chosen a constant stepsize. After only 400 steps, the multicast rate converges within $5 \%$ of the optimal value. In order to study the impact of different choices of the stepsize on the convergence of the algorithm, we have run simulations with different stepsizes. We found that the smaller the stepsize, the slower the convergence and the closer to the optimal, which is a general characteristic of any gradient based method. So, there is a tradeoff between convergence speed and optimality.

From Fig. 3 (b), compared with Fig. 3 (a), all rates indeed converge to the optimal values instead of only a small neighborhood. Compared with Fig. 3 (b), all rates in Fig. 3 (c) not only converge to the optimal values but converge at a speed much faster than the second algorithm. From these results, we conclude that the first and the third algorithms are preferable as they have faster convergence rate.

\section{CONCLUSION}

We have proposed three rate control algorithms for multipath based rate control for multicasting with network coding. Compared with the algorithms in [2], the multipath based multicast rate control algorithms are more complex. Each source needs to update the rates of all paths for each receiver. On the other hand, it is easier to find a minimum cost set of paths compared to trees, and the multipath formulation is more flexible and can result in better performance. The distributed nature of our algorithms allows them to be suitable for a variety of network settings and applications.

\section{REFERENCES}

[1] R. Ahlswede, N. Cai, S. Y. R. Li, and R. W. Yeung, "Network information flow," IEEE Trans. Inform. Theory, vol. 46, no. 4, pp. 1204-1216, Jul. 2000.

[2] L. Chen, T. Ho, S. H. Low, M. Chiang, and J. C. Doyle, "Rate control for multicast with network coding," in Proc. of IEEE Infocom, 2007.

[3] T. Cui and T. Ho, "Minimum cost network coding with integer flows," in Proc. of IEEE ISIT, 2007.

[4] F. Kelly, A. Maulloo, and D. Tan, "Rate control in communication networks: shadow prices, proportional fairness and stability," J. Oper. Res. Soc., vol. 49, no. 3, pp. 237-252, March 1998.

[5] S. H. Low and D. E. Lapsley, "Optimization flow control-I: Basic algorithm and convergence," IEEE/ACM Trans. Networking, vol. 7, no. 6, pp. 861-874, Dec. 1999.

[6] H. Sherali and C. Gyunghyun, "Recovery of primal solutions when using subgradient optimization methods to solve Lagrangian duals of linear programs," Oper. Res. Lett., vol. 19, pp. 105-113, Sept. 1996.

[7] D. P. Bertsekas and R. Gallager, Data Networks. Prentice Hall, 1992.

[8] K. Kar, S. Sarkar, and L. Tassiulas, "Optimization based rate control for multirate multicast sessions," in Proc. of Infocom, Mar. 2001.

[9] S. Deb and R. Srikant, "Congestion control for fair resource allocation in networks with multicast flows," IEEE/ACM Trans. Networking, vol. 12, no. 2, pp. 274-285, April 2004.

[10] D. Lun, N. Ratnakar, M. Médard, R. Koetter, D. Karger, T. Ho, E. Ahmed, and F. Zhao, "Minimum-cost multicast over coded packet networks," IEEE Trans. Inform. Theory, vol. 52, pp. 2608-2623, 2006.

[11] Y. Wu and S.-Y. Kung, "Distributed utility maximization for network coding based multicasting: A shortest path approach," IEEE J. Select. Areas Commun., vol. 24, no. 8, pp. 1475-1488, Aug. 2006.

[12] T. Ho and H. Viswanathan, "Dynamic algorithms for multicast with intra-session network coding," in Proc. of Allerton Conference on Communication, Control, and Computing, Sept. 2005.

[13] W.-H. Wang, M. Palaniswami, and S. H. Low, "Optimal flow control and routing in multi-path networks," Performance Evaluation, vol. 52, no. 2-3, pp. 119-132, April 2003.

[14] X. Lin and N. B. Shroff, "Utility maximization for communication networks with multipath routing," IEEE Trans. Automat. Contr., vol. 51, no. 5, pp. 766-781, May 2006.

[15] T. Ho, M. Médard, R. Koetter, D. Karger, M. Effros, J. Shi, and B. Leong, "A random linear network coding approach to multicast," IEEE Trans. Inform. Theory, vol. 52, pp. 4413-4430, Oct. 2006.

[16] D.Waitzman, S. Deering, and C. Partridge, "Distance vector multicast routing protocol," RFC 1075, Nov.1988.

[17] T. Cui, L. Chen, and T. Ho, "Optimization based rate control for multicast with network coding: A multipath formulation," Caltech, Tech. Rep., March 2007. 